\title{
Aspects of a Product Model Supporting Apparel Virtual Enterprises
}

Peter O. Denno

U.S. DEPARTMENT OF COMMERCE Technology Administration National Institute of Standards and Technology

Gaithersburg, MD 20899 



\section{NISTIR 5821}

\section{Aspects of a Product Model Supporting Apparel Virtual Enterprises}

\section{Peter O. Denno}

U.S. DEPARTMENT OF COMMERCE Technology Administration

National Institute of Standards and Technology

Gaithersburg, MD 20899

April 1996

U.S. DEPARTMENT OF COMMERCE Michael Kantor, Secretary

TECHNOLOGY ADMINISTRATION

Mary L. Good, Under Secretary for Technology

NATIONAL INSTITUTE OF STANDARDS

AND TECHNOLOGY

Arati Prabhakar, Director 



\title{
Aspects of a Product Model Supporting Apparel Virtual Enterprises
}

\author{
Peter Denno \\ pdenno@cme.nist.gov \\ National Institute of Standards and Technology, \\ Gaithersburg, Maryland 20899
}

\begin{abstract}
$\underline{\text { Abstract }}$
Emerging computer technology, including an infrastructure for virtual enterprises and more capable apparel product development software, is providing an opportunity to reinvent how apparel is produced. The development of an information-rich and unambiguous garment product model would help realize this opportunity. This product model would improve the effectiveness of product development software and enhance the industry's ability to distribute work in virtual enterprises. This paper suggests what such a product model should contain, how it can be developed and how it would be utilized throughout the product development life cycle to enable apparel virtual enterprises.
\end{abstract}

\section{Introduction}

The American Apparel Manufacturers Association (AAMA) has defined a standard for the exchange of apparel pattern piece data [AAMA]. This standard has enabled software products from various vendors to interoperate. However, emerging apparel product development systems require more extensive design and product specification information than the current AAMA exchange form provides. These systems would be better served by a standards-based exchange form that allows the systems to communicate the breadth of garment product information that they contain. This new exchange format could also enhance the operation of apparel virtual enterprises, an entrepreneurial-spirited mode of business that is becoming possible as businesses increasingly use computer and Internet technology in the product development cycle.

This paper considers what a new pattern data exchange form, called here the virtual garment prototype (VGP), should contain in order to support emerging apparel product development design software and apparel virtual enterprises. The first section of the paper discusses the old and new information requirements of apparel design systems. The second section outlines the content of the VGP. The third section considers how the apparel industry might find competitive advantage by operating as virtual enterprises; and how the VGP would help in this area. The final section discusses how the technology, methodology and standards developed by ISO STEP (International Organization for Standards, Standard for the Exchange of Product Model Data) can be employed as a foundation for the development of the VGP. 


\section{New Information Requirements}

Design development in apparel includes market forecasting, design, pattern making, grading, piece goods and trim sourcing, and prototype creation (Figure 1). Two sorts of software have assisted in these areas: pattern making systems and product data management (PDM or 'specification') systems. Until recently, pattern making systems have played only a minor role in this effort, providing the storage and manipulation of individual pieces as it relates to the larger design process occurring outside the system. Also, PDM systems were not widely used. Both of these situations have changed recently: newer computer-aided pattern making systems are more than geometry editors, and the use of PDM is now commonplace.

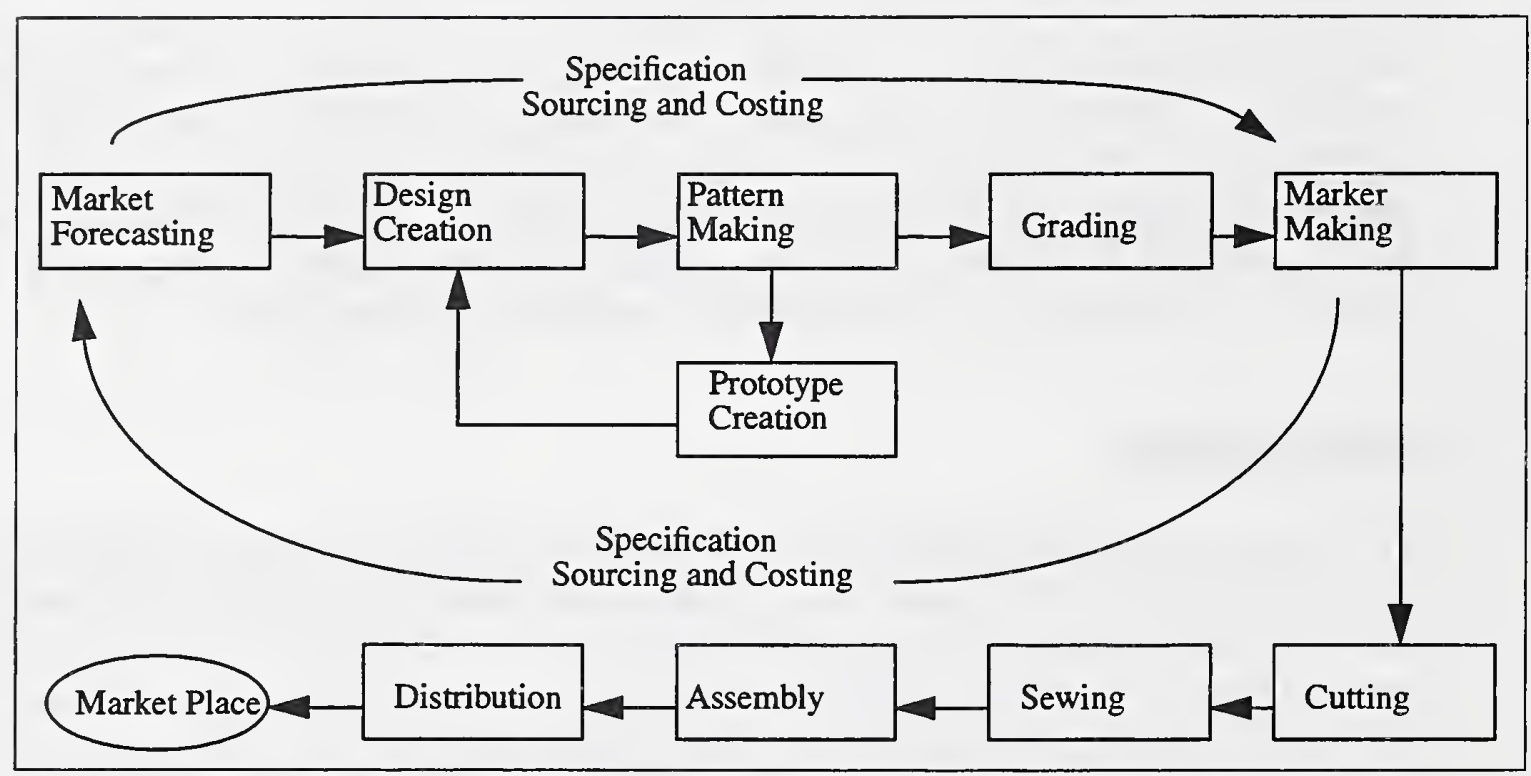

FIGURE 1. Production Activities Showing Cycles of Refinement in Specification and Costing

Pattern making and PDM systems have often been derivative products for vendors whose principle products are marker making systems and cutters. The immediate advantage of computer-aided pattern making is the elimination of the digitizing needed for computerized marker making. The disadvantage of the computer-aided approach is the loss of the 'hands-on feel' of manual pattern making (e.g., tactile smoothness of design lines, sense of life-size proportions of pieces, etc.).

Since pattern making software often originated from the marker making systems, the design system's information model originally lacked design-specific information. The pattern information required for marker making is little more than the grain line and cut line of individual pieces. Design development, of course, encompasses many more activities and information requirements than does marker making.

The advances in pattern making systems are following the trend in geometric design systems in general by providing higher level design abstractions and domain-specific functionality (e.g., manipulation, in concert, of all the pieces composing a seam, generation of 
linings from rough specifications, automated grading). The pattern making system is evolving from an individual piece geometry editor to a system that manipulates assemblies of pieces, thus more closely reflecting the knowledge-intensive work performed by pattern makers, graders and sewers. Instead of requiring that the pattern maker individually modify the geometry of every piece affected by a change, as was previously necessary (for example, to improve fit) the newer systems provide tools to move seams and automatically update every piece associated with the modification.

To make this new functionality possible it is necessary to represent and encode more encompassing information regarding not just the pieces, but the relationship between pieces, and the relationship between the garment and the body. It is necessary to compose seams from pieces, describe linings in relation to complete garments, position fusible under self or shell fabric, and associate fabrics and trims with the garment model.

Finally, information such as seam types, construction details, fabric, and trim specification decreases the ambiguity in what real configuration of the garment the data model describing it actually represents. If enough information is specified, it should be possible for two manufacturers, using nothing but the model, to produce garments that are indistinguishable. ${ }^{1}$

\section{Defining the Virtual Garment Prototype}

Defining a product model is a difficult task. ISO STEP (Standard for the Exchange of Product Model Data) describes useful guidelines to aid in the development of product models such as the VGP [STEP]. Before discussing the STEP method, however, it is useful to consider informally what the model should contain. Therefore, potential VGP modeling features are presented here. In this investigation, two paths to identifying this information have proven useful. One path stems from an analysis of the product development activities. (e.g., market forecasting, conceptual design, pattern making, prototype creation, grading). ${ }^{2}$ This path leads to some obvious and important information requirements, discussed in the following section. A second approach is to consider what work is being performed, or can be performed, by pattern engineering systems. This path can lead to some requirements that are usually left unstated in interactions between people.

1. Models of this level of detail are possible. Discrete part manufacturing has benefited from working to this goal since the early 1970s'. This ability is the essence of the model being free of ambiguity.

2. A scoping of apparel enterprise activities is provided by in [Moncarz]. 


\section{Information Requirements Leading from Activity Analysis}

The activities of market forecasting, design conceptualization, pattern development, prototype creation and sourcing are typically performed in cycles of refinement. Table 1, below, describes some information sources and usage in these activities. In this table information that is recycled for refinement of the product definition is enclosed in square brackets in the Activities Consuming column. In a complete analysis of this type each of the activities would be further decomposed to sub-activities and the information content conveyed between activities would be elaborated upon. The level of information required in one area, construction features, is illustrated in a suit jacket example and seam data model in later sections of the paper.

TABLE 1. Information Producers and Consumers in Product Development Activities

\begin{tabular}{|l|l|l|}
\hline $\begin{array}{l}\text { Activity } \\
\text { Producing }\end{array}$ & Information Produced & Activities Consuming \\
\hline \hline \multirow{3}{*}{$\begin{array}{l}\text { Market } \\
\text { Forecasting }\end{array}$} & market trend & design, sourcing \\
\cline { 2 - 3 } & style concept & design \\
\cline { 2 - 3 } & cost constraints & sourcing \\
\hline \multirow{3}{*}{ Design } & style development & pattern making, [market forecasting] \\
\cline { 2 - 3 } & selection of trims & sourcing \\
\hline \multirow{3}{*}{ Pattern Making } & pieces & prototypes, [market forecasting, design] \\
\cline { 2 - 3 } & piece geometry (e.g., cut lines, sew lines) & grading, sewing \\
\cline { 2 - 3 } & construction features (e.g., seams) & grading, sewing \\
\cline { 2 - 3 } & style features (e.g. folds, fullness features) & grading, sewing, [design] \\
\hline Grading & graded pieces & marker making \\
\hline
\end{tabular}

\section{Information Requirements of Pattern Engineering Systems}

In this paper the term pattern engineering system refers collectively to product data management (PDM), and pattern development systems. Grouping these systems together is justified from the standpoint of fulfilling their similar information requirements. Commercial products that are clearly focused on either pattern development or garment specification are better served by a single data exchange specification that addresses both than by an exchange specification that addresses only one of these systems. This is because there is significant interaction possible between the data from each system: specification can affect pattern pieces (e.g., seam type can effect seam allowance); and pattern piece can affect specification (e.g., piece size can determine garment measurements). A goal of the virtual garment prototype is to provide an exchange form for pattern engineering systems.

It is possible to identify some of the content of the VGP by considering how software would employ it. This approach has the advantage of not assuming too much; where people often see obvious relationships (e.g., sleeve cap and armhole go together) computers need a formal relationship defined. The list below enumerates some of the higher-level 
abstractions that are absent from existing apparel pattern exchange standards. The problem facing pattern engineering systems in using these exchange forms is the need to reestablish these higher-level abstractions and relationships after receiving the exchanged data. $^{1}$

- Automated grading: requires knowledge of seams, fullness, assembly, orientation and placement cues between body and garment, target garment dimensions, dimensioning on assemblies.

- Pattern development tools: requires piece relationships across multiple pieces that react to modifications in concert. This, in turn, requires knowledge of seams, assembly, fullness features, orientation and placement cues, and pattern making techniques.

- Generation of cut line from sew line: requires knowledge of seam properties, stitch properties, and fabric properties.

- Generation of linings and fusibles: requires design specifications, knowledge of shell assembly, as well as the requirements of pattern development tools above.

- Draping visualization: requires knowledge of assembly, and material properties for shell, fusibles and lining, orientation and placement cues between the body and garment.

- Pattern adjustments for alternative fabrics: requires knowledge of assembly and material properties for original and alternative fabrics, pattern making techniques.

- Costing (estimating marker utilization, and other downstream considerations): requires reference to vendor data for fabrics, trims and assembly information.

\section{An Example: A Suit Jacket Sleeve and Front Panel}

In the previous section potentially complicated constructs were referred to with terms such as 'properties of' or 'knowledge of'. This section develops a few of these constructs further. To illustrate how some of the suggested features of the VGP are integrated and employed, an example is provided below. Figure 2 shows the partial detail of information describing a suit jacket sleeve and front panel. The figure shows essential construction, dimensioning annotation and body landmarks for use in pattern making, draping simulation and grading. The example does not detail the collar, pockets, sew line, notches or sewing. By identifying both common seams and coincident points as shown in the figure, the seam ease inherent in the seam is represented. Body landmarks on the pieces help to identify the relationship between the garment and the body for use in grading and draping.

Although the construction details of this jacket are mostly typical, the detail shown is not sufficient for computerized reconstruction of the garment. For example, it is not clear at this level of detail whether the pieces overlay or abut at the seams. There are many examples (e.g., pockets commonly employed in outerwear), where only the responsible pattern

1. The pattern information of the virtual garment prototype can be encoded as a flat-pattern model (as is the AAMA model). Although some usages, such as draping simulation, are 3-dimensional, the creation of the 3-dimensional shape from flat-pattern pieces is possible using construction information that describes seams, fullness and design features, etc. Construction information reduces ambiguity in the model. 
maker would understand the construction from individual, unannotated pattern pieces. In these cases either a real prototype or VGP is essential.

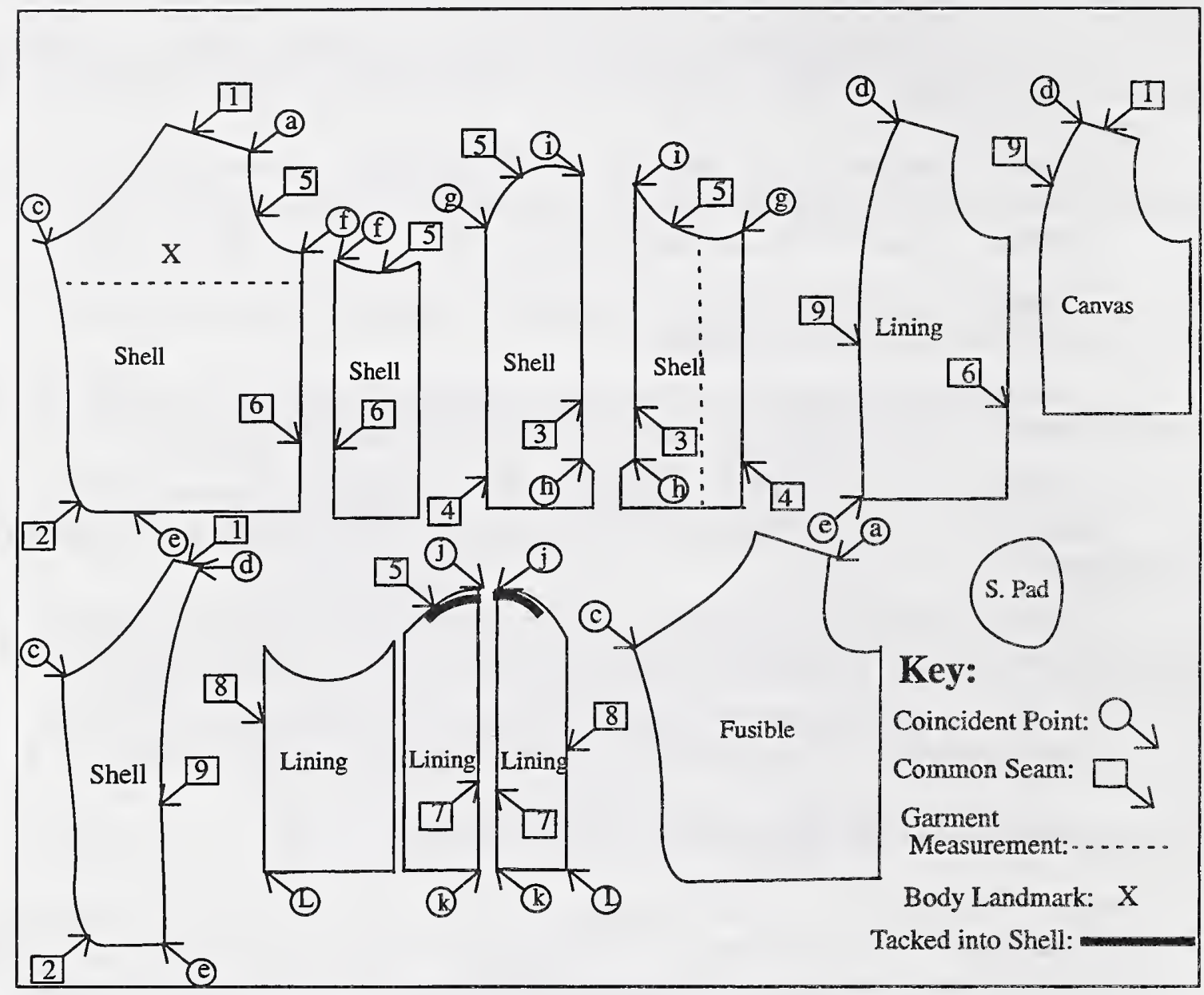

FIGURE 2. Construction Detail of a Suit Jacket Sleeve and Front Panel

Figure 3 (next page) is a graphical representation of a data model defining a seam. This figure is an elaboration of the information describing seams found in Figure 2. The common seams and coincident points of Figure 2 are represented here by Sewn Seam and Mating End P $t$ data entities respectively. The figure illustrates the complexity of information necessary to unambiguously describe a seam. The representation is intended to allow description of seam ease, and different stitch types across multiple pieces. Similar levels of complexity are required to describe body landmarks, vendors, grading dimensions, and fullness features, etc. This figure uses a graphical representation of the data specification language used in STEP product data modeling. Such data specifications are an end product of the collaborative efforts between apparel and STEP data modeling experts. With these representations in hand, software tools can be applied to automaticaliy produce software to exchange the product data. This software can be included in pattern engineering and product data modeling software products, making it possible for them to interoperate with each other. 


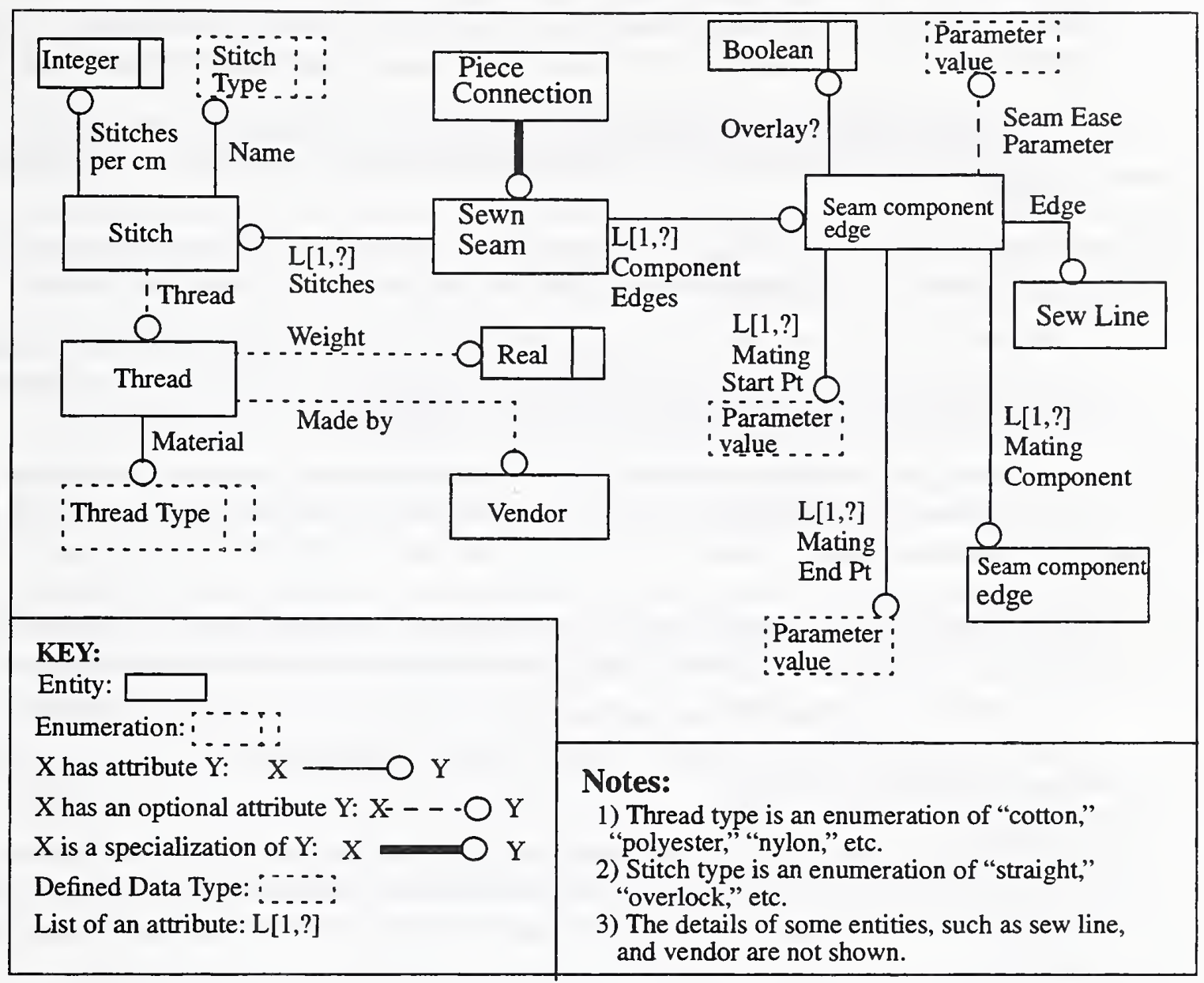

FIGURE 3. An EXPRESS-G Model of a Seam

\section{The Virtual Garment Prototype in Apparel Virtual Enterprises}

\section{Industrial Virtual Enterprises}

Having suggested the content of the VGP, this section discusses the purposes it would serve among apparel virtual enterprises, a entrepreneurial-spirited mode of business that could change the way the industry operates.

Virtual enterprises (VEs) are currently the focus of much attention and research both in apparel [AMTEX] and more generaily [Davidow, NIIIP]. The National Industrial Information Infrastructure Protocols (NIIIP) is a 30 month, $\$ 30$ million Technology Reinvestment Project developing infrastructure and standards to enable virtual enterprises. NIIIP defines a virtual enterprise as "a temporary consortium of individuals, often crossing company boundaries, who come together quickly with the goal of exploiting fast-changing opportunities. Within the virtual enterprise, its members share costs and skills, each contributing their core competencies. VEs are formed without regard to size, organizational, or technical boundaries in order to provide timely and competitive products and services." 
The Internet has, more than any other technology, enabled the emergence of virtual enterprises. However, a virtual enterprise is more than just business use of the Internet. Virtual enterprise technology provides the VE temporary consortium with the following services:

- Work flow management: participants in a VE rely on VE software to coordinate schedules, present work tasks to individuals at the proper time and coordinate data access.

- Creation/Dissolution: VE software provides the ability to select participating companies, define roles and schedules and clean up after the work is complete.

- Security: VE software protects intellectual assets from malicious outsiders and industrial espionage. Protection might even include insulating members from each other's aspects of business which are not part of the collaboration.

- Access to product data: particularly in an industrial virtual enterprise, where the goal is to produce a manufactured product, the members must be able to communicate and share product data.

The above services are essential qualities of collaborative manufacturing of any sort. In particular, VEs could provide the apparel industry with some significant advantages: ${ }^{1}$.

- The virtual enterprise can provide the manufacturer with flexibility in identifying contractors, suppliers and facilities for the production of its products (commonly referred to as an agile supply chain).

- Conversely, a manufacturer with excess capacity may use the VE infrastructure to become a contractor. For example, a manufacturer with a predictable slump between seasons in its cutting and sewing facility might offer cutting and sewing to other enterprises. $^{2}$

- The virtual enterprise provides the small business and those possessing sought after skills with a market for their services. This is particularly useful to apparel manufacturers in the United States, where pattern makers are in high demand.

To serve these purposes the VGP (and product models in general) should possess the following qualities: (1) the information must interoperate in an environment characterized by heterogeneous computer systems and various software applications (the VE environment); (2) the product model should strive to eliminate ambiguity pertaining to the product's actual characteristics; (3) the product model should strive to eliminate the reconstruction of work performed previously in the development process. These ideas are discussed below.

1. Some of these ideas are already being tested in the AMTEX Demand Activated Manufacturing Architecture [AMTEX]

2. Of course, this is not a new idea, but by possessing interoperable, information-rich descriptions of products buyers and suppliers can more easily assess the feasibility of collaboration. 


\section{Barriers to Work Distribution}

Today, in large apparel producing cities, there are companies that specialize in garment grading for a diverse group of local customers. Apparel virtual enterprises would make it possible to extend this distribution of work into more tasks of the design cycle and to provide a global market for customers and suppliers of the work. To achieve this, the essential product information must be interoperable, distributed geographically, and shared temporally, as the design process proceeds.

Distributing the design of apparel is a difficult task because apparel design typically requires close cooperation between the various contributors over a very short schedule. As illustrated in figure 1, product development tasks are interleaved with costing and sourcing activities. The process of determining a production design is one of iterative refinement, where the principle design tasks are influenced by current market and cost information. The design process requires the cooperation of a team with very diverse skills: merchandisers forecast the market for an idea; designers develop conceptual designs; pattern makers make patterns from conceptual sketches, graders reproduce patterns in various sizes, and sourcing buyers find suppliers for the product's piece goods and trims.

Although the diversity of skills required argues for distribution into guilds (i.e., pattern making, grading or sourcing are out-sourced to firms with expert ability), the problem of communicating information into each task is a barrier against this happening. For example, when a team consisting of merchandiser/designer and pattern maker decide that the sleeve of a garment has too much fullness at the armhole, the pattern maker must modify the design. If the exchange form only encodes individual piece geometry, integral characteristics of the garment construction must be recovered. In order to make the modification, the pattern maker must first mentally reconstruct the sleeve, front, back, lining, fusible and perhaps shoulder pad placement and consider the fabric properties involved before judging how to modify the garment. To do this mental reconstruction with ease the pattern maker must have either recently worked on the pattern (arguing against flexibility in who does the work) or have a prototype in hand (arguing against operation as a virtual enterprise). Mental reconstruction, which also occurs in grading, is a consequence of the necessary information being absent from the exchange form.

In summary, storing the relationships of an information-rich garment product model will enhance the ability of people to quickly grasp the significant structure of the problem and will eliminate the introduction of errors that would occur were it necessary to reconstruct and make assumptions about the product configuration. These attributes are essential to operation in a virtual enterprise environment.

\section{Methodology for Development of the Product Model}

The complexity of the VGP product model calls for its development by a cross-trained team of industry and information modeling experts. A methodology for the development 
of product models has been defined by the Standard for the Exchange of Product Model Data (STEP) [Fowler]. This section introduces STEP and the STEP architecture.

ISO (International Organization for Standards) TC184/SC4, is a technical committee that is developing an international standard, ISO-10303 (commonly called STEP) for representing product model data and its exchange. The STEP community comprises 26 national member bodies. STEP development is industry driven, rather than driven by software vendors. The initial release of STEP (December, 1994) includes the definition of an information modeling language, information resources which are general enough to support multiple industries, and specific application protocols for 3-Dimensional design and drafting. Many other STEP application protocol are approaching international standards status, including such diverse areas as ship construction and NC cut-path planning.

\section{STEP Architecture}

In STEP terminology, a virtual garment prototype product model would be described by an application protocol (AP). An AP is a computer and human readable specification document written using the EXPRESS information modeling language [Schenck]. The application protocol defines concepts or entities containing typed attributes. ${ }^{1}$ The AP also contains rules and relationships governing types and values of attributes, the cardinality of sets of entities, etc. These rules reflect the semantics of the real-world artifact being modeled. For example, a rule might require that seam entities reference sew-line entities, not cut-line entities. These rules are defined to ensure the fidelity of the model to the intentions of the industry experts involved in its definition and the application programs that exchange data by it. (For example, applications that exchange information in the form of the AP promise to describe seams by reference to sew-lines, not cut-lines.) Such rules are the basis of software interoperability.

The application protocol, even in an entirely new industrial area for STEP application, relies on pre-defined basic building block entities called integrated resources that have been established as commonly useful to the description of artifacts (e.g., bezier curves, release approvals, etc.) [Barnard]. Domain specific concepts, such as the sew line can be produced by specializing a similar concept in the integrated resources. Hence, due to integrated resources, it is not necessary for VGP developers to define by themselves the substantial foundation of concepts that would be necessary without integrated generic resources. To date these integrated resources have been rich enough to support, with only minor extension, a diverse set of applications.

Similarly, STEP defines application interpreted constructs for the consistent representation of commonly used concepts across domains. Application interpreted constructs can be thought of as a library containing a set of concepts that satisfy a specific purpose. Because the same application interpreted construct can be found in applications across disciplines (e.g., a pattern development system and a production scheduling system might share a construct that indicates that a pattern is approved for marker making) they provide

1. Entities can be thought of as corresponding to objects in programming languages. Entities are identified by rectangles in the EXPRESS-G diagram of figure 3. 
a degree of interoperability between these systems. Application interpreted constructs are a means for various systems to communicate common concepts.

\section{Conclusion}

The garment industry is perhaps the industry that could most benefit by operating as virtual enterprises. Unlike the aerospace and automotive industries, the garment industry does not rely on sophisticated, proprietary manufacturing processes that tend to keep finished product manufacturing concentrated in a few companies. Sewing is largely a commodity, and sewing requirements are easily communicated. The garment industry relies on many globally distributed contractors for production. What is missing here is only agility in the distribution of work among this large pool of contractors.

Further, apparel product development requires the cooperation of individuals with a very diverse skill set. Some of these skills, particularly pattern making and grading are continually in short supply in the United States. Some of this shortage will be met by better pattern engineering systems; some shortage could be relieved were the people with these skills able to reach a global market for their work, that is, were apparel virtual enterprises common.

As a means to enable more agile production, the advantage of apparel virtual enterprises is even more obvious. Some segments of the industry experience huge fluctuations in product development effort, tracking the transition from one fashion season to the next. By operating in virtual enterprises, other segments of the industry could more easily utilize the product development capacity of this segment during its slow period.

Finally, there is the largely unexplored domestic market for custom clothing. The efficient manufacture of custom clothing requires agility in manufacturing and intelligence in product models. Automated grading for custom fit, for example, is possible only when body measurements can be related back to garment dimensions, to piece constructions and finally to individual pieces. This requires an information model that contains the highlevel abstractions such as garment dimensions and the relationships that bridge levels of abstractions down to primitive geometry.

Both apparel virtual enterprises and pattern engineering systems require a standards-based product model that is information-rich in the high level abstractions of the product development process. The product model must communicate the design unambiguously. It must not require that product developers re-establish these high level abstractions and relationships when receiving the model. The information must be encoded in the exchange form.

This paper suggests what an information-rich garment product model should contain. Further it proposes to leverage STEP technology and methodology which is well suited for its development. The Ready-to-Wear pattern making STEP AP [Lee], may serve as a starting point for the development of the VGP. 


\section{Acknowledgments}

This paper would not be possible without the help of KaMing Tam and the many discussions I enjoyed with her and her colleagues at London Fog and elsewhere. Thanks are due also to Mary Mitchell and the apparel project people at NIST (Tina Lee, Howard Moncarz and Jeane Ford).

\section{References}

[AAMA] American National Standard for Pattern Data Interchange - Data Format, ANSUAAMA-292, American National Standards Institute, Inc., September 3, 1993.

[AMTEX] The American Textile Partnership, DAMA Homepage, http://dama.tis.llnl.gov/.

[Barnard] Barnard Feeney, Allison. Gilbert, M., and Yang, Y. Guidelines for AIM Development, ISO TC184/SC4 N304, in print.

[Davidow] Davidow, William, H., and Malone, Michael, S., The Virtual Corporation: Structuring and revitalizing the corporation for the 21st century, HarperBusiness 1992.

[Fowler] Fowler, J., STEP for Data Management, Exchange and Sharing, Technology Appraisals Ltd., 1995.

[Lee] Lee, -Y., Tina., Extensions of the Prototype Application Protocol for Ready-to-Wear Apparel Pattern Making, National Institute of Standards and Technology, NISTIR 5727, October, 1995.

[Moncarz] Moncarz, Howard, T., and Lee, Y., T., Report on Scoping the Apparel Manufacturing Enterprise, International Journal of Clothing Science and Technology, MCB University Press, issue 4/5, 1993.

[NIIIP] NIIIP Homepage, http://www.niiip.org/.

[Schenck] Schenck, Douglas and Wilson, Peter. Information Modeling: The EXPRESS Way, Oxford University Press, 1994. 

